\title{
Propranolol Ameliorates the Development of Portal-Systemic Shunting in a Chronic Murine Schistosomiasis Model of Portal Hypertension
}

\author{
S. K. Sarin, ${ }^{\star}$ R. J. Groszmann, ${ }^{\star}$ P. G. Mosca, ${ }^{\star}$ M. Rojkind, ${ }^{\star}$ M. J. Stadecker, ${ }^{3}$ R. Bhatnagar, ${ }^{3}$ A. Reuben, ${ }^{\star}$ and Y. Dayal \\ Hepatic Hemodynamic Laboratory, Veterans Administration Medical Center, West Haven, Connecticut 06516, and Department of \\ Medicine, Yale University School of Medicine, New Haven, Connecticut*; Liver Research Center, Albert Einstein College of Medicine, \\ New York, New York ${ }^{\ddagger}$; and Department of Pathology, Tufts University Medical School, Boston, Massachusetts ${ }^{\S}$
}

\begin{abstract}
We investigated the role of early portal hypotensive pharmacotherapy in preventing the development of portal-systemic shunting in a portal hypertensive model of chronic murine schistosomiasis induced by infecting $\mathrm{C} 3 \mathrm{H}$ mice with 60 cercariae of Schistosoma mansoni. Propranolol was administered in drinking water to 20 animals for a period of 6 wk at a dose of 10 $\mathrm{mg} \cdot \mathrm{kg}^{-1} \mathrm{~d}^{-1}$, starting at 5 wk of schistosomal infection. 32 agematched mice with chronic schistosomal infection served as controls. All animals were studied 11 wk after the infection. Compared with controls the portal pressure $(10.8 \pm 0.40$ $\mathrm{mmHg})$ was significantly lower $(P<0.001)$ in the propranololtreated animals $(7.9 \pm 0.80 \mathrm{mmHg})$. Portal-systemic shunting was decreased by $79 \%$, from $12.2 \pm 3.34 \%$ in controls to $\mathbf{2 . 5} \pm \mathbf{0 . 9 9} \%$ in the propranolol group $(P<0.05)$. Portal venous inflow was reduced by $38 \%$ in the propranolol treated animals $(2.50 \pm 0.73 \mathrm{ml} / \mathrm{min} ; n=6)$ compared with controls $(4.00 \pm 0.34$ $\mathrm{ml} / \mathrm{min} ; n=8 ; P<0.05)$. The worm burden, the granulomatous reaction, the collagen content of the liver, and the serum bile acid levels were not significantly different between the two groups of animals. These results demonstrate that in chronic liver disease induced by schistosomiasis, the development of portal-systemic shunting can be decreased or prevented by the reduction of flow and pressure in the portal system. (J. Clin. Invest. 1991. 87:1032-1036.) Key words: fibrosis • collagen • bile acids - portal-collateral circulation - hemodynamics in chronic liver diseases
\end{abstract}

\section{Introduction}

Nonselective beta-adrenergic blocking agents such as propranolol are used, with variable success, to treat established portal hypertension. These agents decrease portal pressure by reducing the increased portal venous inflow (1-3). At present, it is unknown whether treatment with beta-adrenergic blocker, used early in the development of portal hypertension, can prevent the development of the most serious portal hypertensive complication, i.e., portal-systemic shunting.

Address correspondence and reprint requests to Dr. Robert J. Groszman, Hepatic/Hemodynamic Laboratory 111/J, VA Medical Center, West Haven, CT 06516. Dr. Sarin's present address is Department of Gastroenterology, G. B. Pant Hospital, New Delhi 110002, India.

Received for publication 26 March 1990 and in revised form 3 October 1990

\section{J. Clin. Invest.}

(C) The American Society for Clinical Investigation, Inc.

0021-9738/91/03/1032/05 \$2.00

Volume 87, March 1991, 1032-1036
Chronic murine schistosomiasis provides a natural disease model of gradually evolving portal hypertension. The murine model manifests the clinical as well as the histological features of human hepatic schistosomiasis (4), the world's most prevalent chronic liver disease (5). We have already studied the development of portal hypertension and its relationship to the formation of portal-systemic shunting in this model (6). More recently we have observed that, as seen in other models of portal hypertension (7-9), a hyperdynamic syndrome characterized by an increased portal venous inflow also develops in murine schistosomiasis (10). In this study, we investigated whether early therapy with a portal blood flow and pressure-reducing agent, such as propranolol, could ameliorate the development of portal-systemic shunting in the chronic murine schistosomiasis model of portal hypertension (11).

\section{Methods}

Murine model of schistosomiasis. 6-8-wk-old mice of the $\mathrm{C} 3 \mathrm{H}$ strain, (Harlan Sprague Dawley, Inc., Indianapolis, IN) were infected with 60 cercariae of Schistosoma mansoni, Puerto Rico strain. Mice were infected through the shaved abdominal skin using the percutaneous ring method according to a previously described technique (12).

Experimental design. Animals infected with schistosomiasis were divided into two groups, $5 \mathrm{wk}$ after introducing the infection. One group received propranolol and the other served as an untreated control group.

Propranolol administration. DL-Propranolol (Sigma Chemical Co., St. Louis, MO) was dissolved in the drinking water. It was started at 5 wk of schistosomal infection, animals received an approximate dose of $10 \mathrm{mg} \cdot \mathrm{kg}^{-1} \cdot \mathrm{d}^{-1}$, for a period of $6 \mathrm{wk}$. Both groups of animals were studied at $11 \mathrm{wk}$ of schistosomal infection.

Hemodynamic studies. Animals were coded, weighed, and anesthetized with nembutal (pentobarbital sodium, $0.05 \mathrm{mg} / \mathrm{g}$ body wt). The anesthetized animal was placed in the supine position. Temperature was maintained at $37 \pm 0.5^{\circ} \mathrm{C}$ by a thermostatic mattress (Harvard Apparatus Co., Inc., S. Natick, MA) and monitored by a rectal probe. Subdermal electrodes (E-2 electrodes; Grass Instrument Co., Quincy, MA) were inserted in the tail and palms and connected to a cardiotachometer (Grass Instrument Co.) for continuous recording of heart rate and electrocardiogram.

Splanchnic hemodynamics were measured by the reference sample technique according to methods previously described $(13,14)$. Briefly, the right carotid and the left femoral artery were exposed and cannulated. The carotid catheter which was advanced under arterial pressure monitoring to the base of the carotid artery was used for injection of ${ }^{103} \mathrm{Ru}$-radiolabeled microspheres ( $16.5 \pm 0.1 \mu \mathrm{m}$; New England Nuclear, Boston, MA). The right femoral artery catheter was employed for obtaining the reference blood sample. We have earlier validated the technique of injecting radioactive microspheres into the carotid artery (14).

Each catheter was connected to a Statham P-23-Db strain-gauge transducer (Statham, Oxnard, CA) and recordings of arterial pressure 
were made on a Grass model 7D inscription recorder (Grass Instrument Co.). External zero reference limit was placed at the midportion of the mouse.

Organ blood flow (milliliter per minute), the arterial blood flow of each organ, was calculated as previously described $(7,14)$. Portal venous inflow is the sum of blood flow to the stomach, spleen, small and large intestines, pancreas, and mesentery. Because the venous outflow of each of these organs goes into the portal venous system, portal venous inflow represents the total splanchnic arterial inflow entering the portal venous system. Portal venous inflow in this study has been expressed in milliliters per minute and in milliliters per minute per $100 \mathrm{~g}$ body wt.

Portal pressure. A small parasagittal incision $(1.0-2.0 \mathrm{~cm})$ at the level of the left anterior axillary line was made in the skin over the left upper abdominal quadrant and the muscles were gently separated with forceps. The spleen was exposed and covered with warm, saline-moistened gauze. Care was taken to avoid any unnecessary manipulation of the spleen. The spleen was impaled with PE10 polyethylene tubing connected to a Statham P-23-Db strain gauge transducer. Continuous tracings of the splenic pulp pressure, were recorded on a Grass model 7D inscription recorder. We have earlier observed that intrasplenic pressure closely correlates with portal pressure obtained by puncturing the superior mesenteric vein (8).

Portal-systemic shunting. Spleno-portal-systemic shunting was estimated by adapting a technique previously described (13). A splenic pulp injection of $\sim 30,000{ }^{141} \mathrm{Ce}$-labeled microspheres, $16.5 \pm 0.1 \mu \mathrm{m}$ in diameter, mixed in $0.1 \mathrm{cc}$ of $0.9 \% \mathrm{NaCl}$ was made over $20 \mathrm{~s}$. The injection site was sealed with cyanoacrylate glue. The lungs and liver were removed, cleaned, and weighed. The radioactivity of each organ was determined in a gamma counter (Packard 9042; Packard Instruments Co., Inc., Downers Grove, IL). The extent of portal-systemic shunting was calculated from the radioactivities in lungs and liver, applying a previously described formula (13). Standards for ${ }^{103} \mathrm{Ru}$ and ${ }^{141} \mathrm{Ce}$ were used to allow for the spillover of ${ }^{103} \mathrm{Ru}$ into the ${ }^{141} \mathrm{Ce}$ channel.

Assessment of worm burden. After determination of portal pressure and portal-systemic shunting, the animals were killed. The worm burden was determined by perfusion of the portal-mesenteric venous system (12). A careful search for additional worms was made by stripping and inspecting the mesentery and hepatic hilum.

Quantitation of granulomatous liver disease. Representative samples of the liver were fixed in $10 \%$ neutral formalin and processed for light microscopy. The area of the liver involved by the granulomas was quantitated by $R$. Bhatnagar, under code, by means of computer assisted morphometric analysis (6).

Quantitation of liver collagen. Measurement of liver collagen and protein content in tissue sections prepared for light microscopy was performed blindly, on coded samples, according to the method of Lopez-De-Leon and Rojkind (15).

Bile acid determination. Nonfasting arterial blood samples were obtained after the completion of the hemodynamic studies to determine the total serum bile acid level (16).

Statistical analysis. All the observations are reported as mean \pm SEM. The significance of the differences between controls and the propranolol-treated group were estimated by the unpaired Student's $t$ test, the Mann-Whitney U test, or the Chi-square test as appropriate. Normal values for mice systemic and splanchnic hemodynamics have been previously reported by our laboratory $(6,14)$.

\section{Results}

A total of 52 mice infected with $S$. mansoni for 11 wk were studied, i.e., 32 controls and 20 propranolol-treated animals. Mean body weight of the controls $(28.43 \pm 0.48 \mathrm{~g})$ was not different from the propranolol-treated animals $(27.49 \pm 0.52 \mathrm{~g})$. There was also no significant difference in the daily water intake of the animals in the two groups.

\section{Systemic hemodynamics}

As compared with controls $(554 \pm 15 \mathrm{bpm})$, the mean heart rate was significantly reduced in the propranolol-treated animals (493 \pm 16 bpm; $P<0.05)$ (Fig. 1). Similarly, the mean arterial pressure was significantly decreased in the propranolol treated animals (70.31 \pm 5.49 vs. $82.33 \pm 2.72 \mathrm{mmHg} ; P<0.05)$ (Fig. 1$)$.

\section{Portal hemodynamics}

Portal pressure. The mean portal pressure in the propranololtreated group $(7.93 \pm 0.80 \mathrm{mmHg})$ was significantly lower than in the controls $(10.82 \pm 0.40 \mathrm{mmHg} ; P<0.01)$ (Fig. 2).

Portal-systemic shunting. In the propranolol-treated group there was no significant shunting $(<1 \%)$ in $63 \%$ of the animals and $32 \%$ of the animals had portal-systemic shunting of $<10 \%$. In comparison, $44 \%$ of the mice in the untreated group had no shunting $(<1 \%)$ and only $16 \%$ had shunting of $<10 \%$. This indicates that only $5 \%$ of the propranolol-treated animals had shunting of $>10 \%$ vs. $40 \%$ in the untreated animals. The difference between these proportions was statistically significant (Chi-square 7.72; $P<0.05$ ).

All together the mean portal-systemic shunting in the control animals at $11 \mathrm{wk}$ was $12.2 \pm 3.34 \%$; this was reduced to $2.5 \pm 0.99 \%$ in the propranolol-treated animals (Fig. 2) $(P$ $<0.05$ by unpaired $t$ test; $P=0.05$ by Mann-Whitney $U$ test).

Also, in order to evaluate the temporal relationship between propranolol and portal-systemic shunting we studied an additional group of 13 animals, starting oral propranolol at the 7 th wk of infection with $S$. mansoni and terminating the study at $11 \mathrm{wk}$ (4-wk treatment). In these animals the mean extent of portal-systemic shunting $(5.33 \pm 3.03 \%)$ was intermediate between the untreated controls and the animals treated for $6 \mathrm{wk}$. Furthermore, 9 infected untreated mice were studied after 13 wk. In this last group the extent of portal-systemic shunting was higher $(17.31 \pm 7.93 \%)$ than in animals infected for $11 \mathrm{wk}$.

No correlation was observed between portal pressure and portal systemic shunting in either controls or propranololtreated animals. While the average reduction in portal pressure in the propranolol-treated animals was $27 \%$, the reduction in portal-systemic shunting was $79 \%$.

Portal venous inflow. The blood flow in the splanchnic organs was measured, in a subgroup of 8 control and 6 propranolol-treated mice. The portal venous inflow, which represents the total blood flow entering the portal venous system, was $4.00 \pm 0.34 \mathrm{ml} / \mathrm{min}$ in the untreated control mice (Fig. 2). There was a significant reduction $(P<0.05)$ in the portal venous inflow in the propranolol-treated animals $(2.56 \pm 0.73 \mathrm{ml} / \mathrm{min})$. A reduction in portal venous inflow was also observed in the
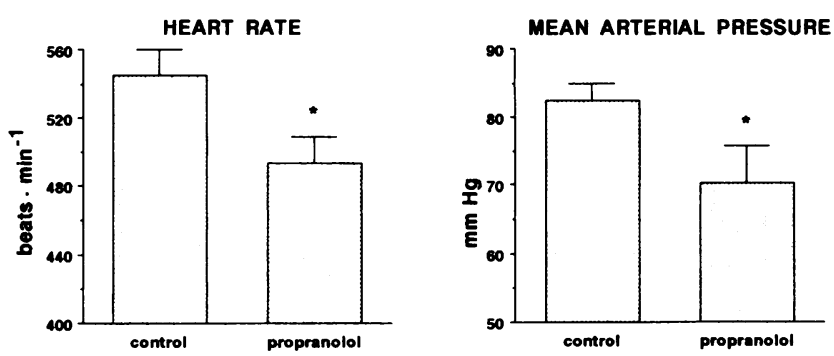

Figure 1. Heart rate and mean arterial pressure in mice after 11 -wk infection with $S$. mansoni. Propranolol was administered for $6 \mathrm{wk}$ at the dose of $10 \mathrm{mg} \cdot \mathrm{kg}^{-1} \cdot \mathrm{d}^{-1} \cdot{ }^{*} P<0.05$. 

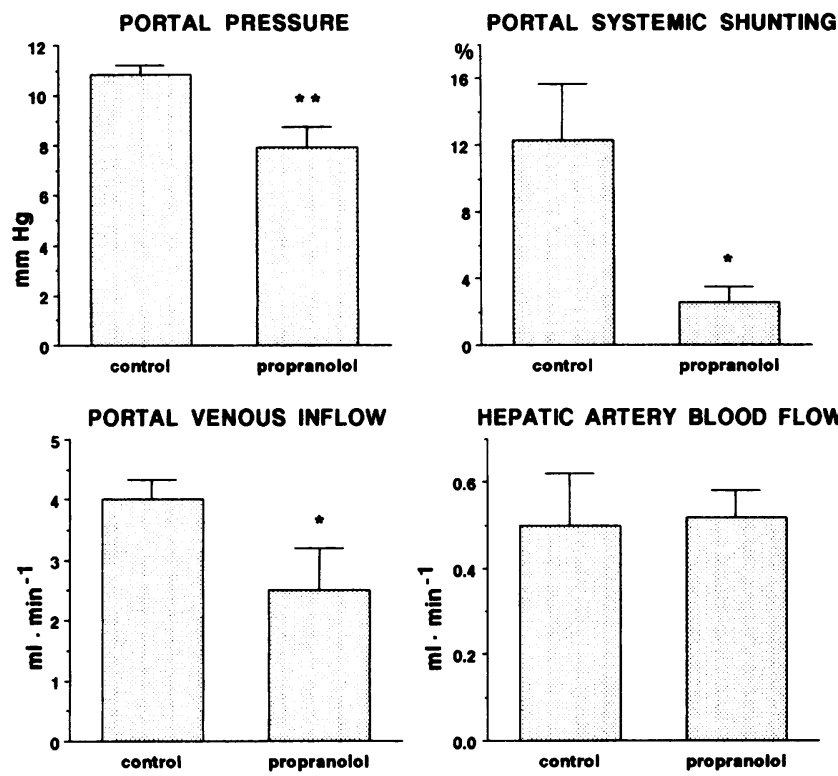

Figure 2. Splanchnic hemodynamics in mice after 11-wk infection with $S$. mansoni. Propranolol induces significant reductions in portal pressure, portal venous inflow, and portal-systemic shunting. No changes in hepatic arterial blood flow are observed. ${ }^{*} P<0.05$; ${ }^{* *} P$ $<0.001$.

propranolol-treated animals when this variable was corrected for body weight $(P=0.05)$. No correlation was seen between portal venous inflow and portal-systemic shunting in controls or in propranolol-treated animals.

Hepatic artery flow. Hepatic arterial blood flow was similar in the propranolol-treated animals $(0.52 \pm 0.06 \mathrm{ml} / \mathrm{min})$ as compared with controls $(0.50 \pm 0.12 \mathrm{ml} / \mathrm{min})$ (Fig. 2$)$. Similarly, when this variable was calculated per gram of liver tissue, there was no significant difference between the two groups.

Liver weight. The mean weight of the liver was similar in the control $(2.45 \pm 0.09 \mathrm{~g})$ and the propranolol-treated animals $(2.59 \pm 0.10 \mathrm{~g})$.

Worm burden. The mean worm burden (total male and female worms) in the untreated controls $(12.72 \pm 1.17)$ was not significantly different than in the propranolol-treated (11.89 \pm 1.46$)$ (Fig. 3).

Quantitation of the granulomatous liver disease. The percentage area of the liver occupied by schistosomal granulomas in the controls $(27.30 \pm 2.03 \%)$ was not significantly different from that in the propranolol-treated group $(27.98 \pm 3.32 \%)$ (Fig. $3)$. No correlation was found between portal pressure or portalsystemic shunting and the degree of granulomatous involvement of the liver.

Liver collagen content. Liver collagen content of infected control mice averaged $32.40 \pm 1.50 \mu \mathrm{g} / \mathrm{mg}$ of protein, which was not significantly different from the value of $32.63 \pm 3.24$ obtained in the propranolol-treated mice (Fig. 3). No significant correlation was observed between the collagen content of the liver and either the portal pressure, the degree of portal systemic-shunting, or the percentage area of the liver occupied by granulomas, in both control and propranolol-treated groups.

Serum bile acids. No significant difference was observed in serum bile acid levels between the treated and control animals: $14.86 \pm 2.35$ vs. $13.07 \pm 2.97 \mu \mathrm{M}$. A strong significant correla- tion between serum bile acid (SBA) level and the degree of portal systemic shunting (PSS) $($ SBA $=7.66+0.54 \times$ PSS; $r$ $=0.80 ; P<0.001)$ was observed only in the control group of mice.

Influence of propranolol on survival. The proportion of animals surviving the $11 \mathrm{wk}$ infection course was not significantly different between control $(51 \%)$ and propranolol-treated animals (59\%) (Fig. 3).

\section{Discussion}

In recent years, several animal models of portal hypertension have been developed for hemodynamic and pharmacological studies. These models include artificial production of portal hypertension by partial constriction of the portal vein, bile duct obstruction, exposure to carbon tetrachloride, or feeding animals with toxic diets $(7,13,17-19,21)$. Chronic murine schistosomiasis, on the other hand, provides a natural disease model of portal hypertension, in which the profile of clinical disease and the hepatic histological changes are quite similar to that seen in humans. Warren and associates have previously demonstrated that schistosomal-infected mice develop hepatosplenomegaly, elevated portal pressure, and esophageal varices (4). In an earlier study $(6,20)$, as well as in this study, we observed that nearly all animals infected with schistosomiasis develop portal hypertension. However, the mouse is the only species that develops portal-systemic shunting $(6,20)$. Also, the schistosomalinfected mouse has been shown to be a relatively homogeneous and predictable model of portal hypertension (6).

Despite the tremendous potential of this murine model, detailed hemodynamic studies have so far not been available. This is probably due to the difficulties encountered in performing vascular catheterization on an animal as small as the mouse. However, we have successfully adapted the radioactive
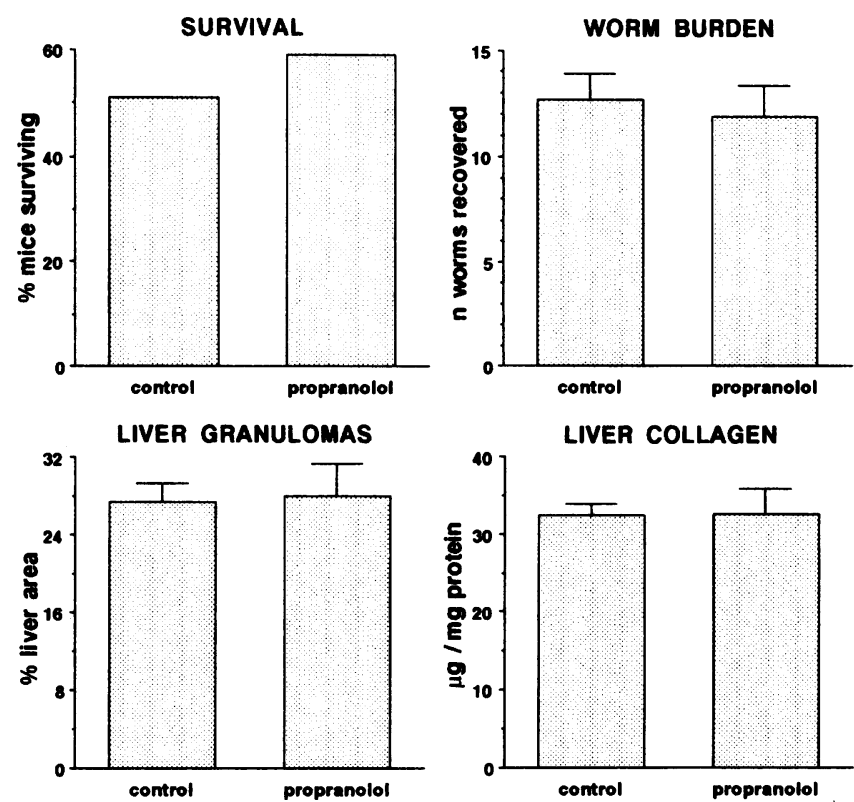

Figure 3. There were no significant differences in the proportion of animal surviving to $11 \mathrm{wk} S$. mansoni infection, the number of adult worms recovered from each animal, the percentage of liver area occupied by granulomas, or the collagen content of the liver, between control and mice treated for $6 \mathrm{wk}$ with propranolol $\left(10 \mathrm{mg} \cdot \mathrm{kg}^{-1} \cdot \mathrm{d}^{-1}\right)$. 
microsphere technique with reference blood sample withdrawal, to study hemodynamics in the mouse model of portal hypertension (14). In this model the elevation in portal pressure is gradual and development of portal-systemic shunting occurs several weeks after the rise in portal pressure (6).

One of the main objectives of this study was to investigate whether a reduction in portal flow and pressure induced by propranolol at an early stage in the disease process, could influence the development of portal systemic shunting. In preliminary studies we had observed that portal pressure begins to rise at 7-9 wk of schistosomal infection in mice (6). Hence, a therapeutic regimen, starting at $5 \mathrm{wk}$ of schistosomal infection was initiated. Our results clearly demonstrate that propranolol therapy, initiated early in the course of schistosomal infection, is effective in reducing portal flow and pressure in the chronic murine schistosomiasis model of portal hypertension. More important is the finding that portal-systemic shunting was also significantly reduced in the propranolol-treated animals. Development of portal-systemic shunting has been shown to be accompanied by a marked reduction in splanchnic arteriolar resistance and an increase in portal venous inflow $(7,9,21)$. The influence of high portal venous inflow in producing and maintaining the portal hypertensive state is now well recognized (8). In addition, it is possible that the increased blood flow through portal-systemic collaterals have an independent (from portal pressure) dilating effect on the collateral vessel (22). Conceptually, therefore, a reduction in portal venous inflow should result in amelioration of portal hypertension and portal-systemic shunting. This hypothesis was supported by the observations of this study. Splanchnic hemodynamic studies performed in a group of controls and propranolol-treated animals showed that propranolol significantly reduced portal venous inflow by $38 \%$. This venous inflow reduction was accompanied by a moderate reduction (of $27 \%$ ) in portal pressure and a dramatic reduction (of $79 \%$ ) in portal-systemic shunting. The discrepancy between the magnitude of reduction in portal pressure and portal-systemic shunting may suggest that the combination of a reduction in flow and pressure in the collateral vessels produces the striking reduction in portal systemic shunting that was observed. Even if the reduction in portal venous inflow may not be sufficient in itself to reduce portal pressure markedly, it may be enough to attenuate the development of portal-systemic shunting. This observation is further supported by the fact that no correlation was seen between portal pressure and portal-systemic shunting. This finding may help to explain why small decreases in portal pressure observed in clinical studies produce important reductions in the incidence of variceal bleeding (23).

Additional information obtained from a group of animals treated with propranolol for a shorter period of time $(4 \mathrm{wk})$, showing an intermediate value for portal-systemic shunting (in between animals treated for $6 \mathrm{wk}$ and untreated animals), suggests that earlier and longer therapy is more effective in preventing the development of portal-systemic shunting. Furthermore, these data demonstrate that the development of portalsystemic shunting is a progressive event and that propranolol changes the natural history of this complication of portal hypertension.

Our results highlight an important concept in the management of portal hypertension, i.e., that of early pharmacological treatment. By reducing the rise in portal pressure and portal venous inflow, one may possibly decrease the development of portal-systemic shunting.

Propranolol, as other nonselective $\beta$-adrenergic blockers, induces a reduction in portal flow and pressure by the combined effect of reducing cardiac output $\left(\beta_{1}\right.$-adrenergic blockade) and splanchnic arterial vasoconstriction $\left(\beta_{2}\right.$-adrenergic blockade) (3).

Could there be other mechanisms, besides reduction in portal venous inflow and pressure to account for the efficacy of propranolol in the decreasing development of portal-systemic shunting? Propranolol did not appear to exert any significant effect on either the worm burden or the granulomatous inflammation in the liver. In addition, we did not observe any difference in liver collagen content between the two groups of animals. In our study, we did not observe any significant effect of propranolol on the collagen content of schistosomal-infected mouse livers, at a time when collagen synthesis and deposition should be maximal (24).

Total serum bile acid levels were equally elevated in both groups of animals (normal value for control mice in Dr. A. Reuben's laboratory is $5.6 \pm 4.4 \mu \mathrm{M}$ ). In the infected mice not treated with propranolol, the highly significant correlation between serum bile acid levels and the degree of portal-systemic shunting suggests that the latter is the main determinant of the increase in serum bile acids as already observed in liver cirrhosis in humans (25) and rats (26). Furthermore, this finding reassures us over the accuracy and interpretation of our measurement of portal-systemic shunting. This relationship is far less strong in the propranolol-treated animals possibly due to a concomitant reduction in the degree of portal-systemic shunting together with a reduction in portal flow (a well known determinant of serum bile acid levels).

The experimental evidence that portal-systemic shunting can be significantly reduced by early propranolol therapy has immense therapeutic potential. Since the development of extensive collaterals and portal-systemic shunting results in gastro-esophageal varices, the possibility of pharmacologically preventing or decreasing development of portal-systemic shunting could represent a major therapeutic goal in the management of portal hypertension and chronic liver disease.

\section{Acknowledgments}

We would like to acknowledge Cindy Erickson for her excellent technical assistance and Rose Allen for the measurement of serum bile acid levels. The editorial and typing assistance of Marge Petrucci is also acknowledged.

This project was supported by a Public Health Service Liver Research Center grant DK-34989, World Health Organization Research grant, and a Veterans Administration Merit Review. Dr. Sarin was a recipient of the John E. Fogarty International Research Fellowship Award. He was supported by the National Institutes of Health grant 9-8420568.

\section{References}

1. Lebrec, D., T. Poignard, P. Hillon, and J. P. Benhamou. 1981. Propranolol for prevention of recurrent gastrointestinal bleeding in patients with cirrhosis. A controlled study. N. Engl. J. Med. 305:1371-1374.

2. Hillon, P., L. Blanchet, and D. Lebrec. 1982. Effect of propranolol on hepatic blood flow in normal and portal hypertensive rats. Clin. Sci. 63:29-32.

3. Kroeger, R. J., and R. J. Groszmann. 1985. Effect of selective blockade of 
$\mathbf{B}_{\mathbf{2}}$ adrenergic receptors on portal and systemic hemodynamics in a portal hypertensive rat model. Gastroenterology. 88:896-900.

4. Warren, K. S., and W. B. DeWitt. 1958. Production of portal hypertension and oesophageal varices in the mouse. Proc. Soc. Exp. Biol. Med. 98:99-101.

5. Dunn, M. A., and R. Kamel. 1981. Hepatic schistosomiasis. Hepatology. 1:653-661.

6. Pons, H. A., J. S. Morgan, M. L. Hutchinson, M. Rojkind, R. J. Groszmann, and M. J. Stadecker. 1989. Resistance to reinfection in experimental murine schistosomiasis; role of the immune system and of porto-hepatic hemodynamics. Am. J. Trop. Med. Hyg. 41:189-197.

7. Vorobioff, J., J. E. Bredfeldt, and R. J. Groszmann. 1984. Increased blood flow through the portal system in cirrhotic rats. Gastroenterology. 87:1120-1126.

8. Sikuler, E., D. Kravetz, and R. J. Groszmann. 1985. Evolution of portal hypertension and mechanisms involved in its maintenance in a rat model. $\mathrm{Am}$. J. Physiol. 248:G618-G625.

9. Benoit, J. N., J. A. Barrowman, S. L. Harper, P. R. Kvietys, and D. N. Granger. 1984. Role of humoral factors in the intestinal hyperemia associated with chronic portal hypertension. Am. J. Physiol. 267:G486-G493.

10. Sarin, S. K., C. Sabbà, P. Mosca, and R. J. Groszmann. 1990. Portal hypertension and hyperdynamic circulation in chronic murine schistosomiasis. Hepatology. In press.

11. Sarin, S. K., M. Stadecker, and R. J. Groszmann. 1989. Propranolol prevents the development of portal-systemic shunting in chronic murine schistosomiasis. Gastroenterology. 96:654a. (Abstr.)

12. Smithers, S. R., and R. J. Terry. 1965. The infection of laboratory hosts with cercariae of Schistosoma mansoni and the recovery of adult worms. Parasitology. 55:695-700.

13. Chojkier, M. S., and R. J. Groszmann. 1981. Measurement of portal-systemic shunting in the rat by using gamma-labelled microspheres. Am. J. Physiol. 240:G371-G375.

14. Sarin, S. K., C. Sabbà, and R. J. Groszmann. Measurement of splanchnic and systemic hemodynamics in mice using a radioactive microsphere technique. Am. J. Physiol. 258:G365-G369.

15. Lopez-De-Leon, A., and M. Rojkind. 1985. A simple micromethod for collagen and total protein determination in formalin fixed paraffin-embedded sections. J. Histochem. Cytochem. 33:737-743.
16. Kobayaski, K., R. Allen, J. R. Bloomer, J., and G. Klatskin. 1979. Enzymatic fluorometry for estimating total serum bile acid concentration. JAMA (J. Am. Med. Assoc.). 241:2043-2045.

17. Ohlsson, E. G., R. B. Rutherford, and J. K. Boitnott. 1970. Changes in portal circulation after biliary obstruction in dogs. Am. J. Surg. 120:16-22.

18. Okudaira, M., H. Kume, M. Ohku, and Y. Aida. 1988. Experimental portal fibrosis induced by chronic feeding of aflatoxin-B1. In Animal Models of Portal Hypertension. S. K. Sarin and N. C. Nayak, editors. Kunj Publishing House, New Delhi, India. 135-141.

19. Okabayashi, A. 1983. Experimental production of non-cirrhotic portal hypertension and splenomegaly with prolonged antigen stimulation. In Idiopathic Portal Hypertension. K. Okuda and M. Omata, editors. University of Tokyo Press, Tokyo. 197-205.

20. Morgan, J. S., R. J. Groszmann, M. Rojkind, and R. Enriquez. 1990. The hemodynamic mechanisms of emerging portal hypertension due to schistosomiasis in the hamster. Hepatology. 11:98-104.

21. Vorobioff, J., J. E. Bredfeldt, and R. J. Groszmann. 1983. Hyperdynamic circulation in portal hypertensive rat model: a primary factor for maintenance of chronic portal hypertension. Am. J. Physiol. 244:G52-G57.

22. Kamiya, A., and T. Togawa. 1980. Adoptive regulation of wall shear stress to flow change in the canine carotid artery. Am. J. Physiol. 230:H14-H21.

23. Groszmann, R. J., J. Bosch, N. Grace, H. O. Conn, G. Garcia-Tsao, M. Navasa, J. Albert, M. Bermann, M. Patrick, and J. Rodes. 1990. Hemodynamic events in a prospective randomized trial of propranolol vs placebo in the prevention of the first variceal hemorrhage. Gastroenterology. 99:1401-1407.

24. El Meneza, S., G. R. Olds, T. F. Kresina, T., and A. A. F. Mahmoud. 1989 Dynamics of hepatic connective tissue matrix constituents during murine Schistosoma mansoni infection. Hepatology. 9:50-56.

25. Ohkubo, H., K. Okuda, S. Lida, K. Ohnishi, S. Ikawa, and I. Makino. 1984. Role of portal and splenic vein shunts and impaired hepatic extraction in the elevated serum bile acids in liver cirrhosis. Gastroenterology. 86:514-520.

26. Reichen, J., B. Egger, N. Ohara, T. B. Zeltner, T. Zysset, and A. Zimmermann. 1988. Determinants of hepatic function in liver cirrhosis in the rat; multivariate analysis. J. Clin. Invest. 82:2069-2076. 